\title{
Gastrointestinal manifestations in Satoyoshi syndrome: a systematic review
}

\author{
Julián Solís-García del Pozo ${ }^{1,2}$ (D) Carlos de Cabo ${ }^{3,4^{*}}$ (D) and Javier Solera ${ }^{1,3}$ (D)
}

\begin{abstract}
Background: Satoyoshi syndrome (SS) [OMIM 600705; ORFHA 3130] is a multisystemic disease with a probable autoimmune basis, whose main symptoms are muscle spasms, alopecia, diarrhea and skeletal alterations. Chronic diarrhea may be severe and result in malnutrition, anemia, growth retardation, cachexia, disability and even death. However, to date, no review of the digestive symptoms has been carried out.

Methods: A search was performed in MEDLINE, Scopus and Web of Science databases. Cases of SS, without language or date restrictions, were recorded. Sixty-seven cases of SS were found up until December 2019. Thirtynine cases described gastrointestinal manifestations.

Results: Chronic diarrhea was the main digestive symptom (92.3\%). Other symptoms such as abdominal pain (15.4\%), nausea (7.7\%) and vomiting (7.7\%), were less frequent. The D-xylose test was positive in 10 out of 12 patients, and 9 out of 13 cases showed a flattened oral glucose tolerance test suggesting carbohydrate malabsorption. Antinuclear antibodies were detected in 8 out of 16 cases. Antibodies to stomach or duodenum tissue lysates were also detected by Western blot. Histological data revealed predominantly lymphoplasmacytic inflammatory infiltrate that can affect any section of the digestive tract. In 6 out of 10 patients, diarrhea improved with a treatment regimen that included corticosteroids. Other treatments, such as methotrexate, carbohydrate restricted diets or otilonium bromide, improved digestive symptoms in isolated patients. Improvement of symptoms up to three years of follow-up has been described. None of the three patients who died had received corticosteroids or immunosuppressants.
\end{abstract}

Conclusion: Chronic diarrhea with malabsorption is one of the most disabling symptoms in SS. The early recognition of this disease is essential for immunosuppressive treatment and a better outcome.

Keywords: Diarrhea, Malabsorption, Rare diseases, Satoyoshi syndrome

\section{Introduction}

Satoyoshi syndrome (SS) [OMIM 600705; ORFHA 3130] is a multisystemic disease, characterized by muscle manifestations in the form of painful spasms or cramps, diarrhea, alopecia, skeletal alterations, growth retardation and menstrual abnormalities [1]. The association of this

\footnotetext{
* Correspondence: carlosd@sescam.jccm.es

${ }^{3}$ Research Department, Neuropsychopharmacology Unit, Complejo

Hospitalario Universitario de Albacete, Albacete, Spain

${ }^{4}$ Hospital General Universitario de Albacete, Unidad de

Neuropsicofarmacología, Edificio de Investigación, 3a planta c/ Hermanos Falcó, 37 E-02008, Albacete, Spain

Full list of author information is available at the end of the article
}

syndrome with other autoimmune pathologies, the detection of autoantibodies in these patients and their response to immunosuppressive treatment, has led to postulate its autoimmune origin [2,3].

Among the most typical features of the syndrome is diarrhea [4]. Chronic diarrhea and malabsorption can lead to malnutrition, weight loss, growth retardation [2], iron- deficiency anemia [5] or hypoproteinemia [6]. Untreated diarrhea has been described as one of the leading causes of morbidity and mortality in patients with SS [1].Diarrhea with signs of malabsorption, weight loss or growth retardation, and the detection of autoantibodies, 
are present in SS and other diseases such as celiac disease, tropical sprue or autoimmune enteropathy.

Previous reviews of SS have focused on muscle symptoms and alopecia [7-10] Our objective in this review is to offer an updated view of the gastrointestinal manifestations and their treatment response in SS.

\section{Main text}

\section{Material and methods}

\section{Search}

All cases were searched in MEDLINE, using the search strategy: "Satoyoshi syndrome" [Supplementary Concept] or "Satoyoshi syndrome" [All Fields], or "komuragaeri disease" [All Fields]) or Satoyoshi [TI]). We also searched in Scopus and the Web of Science, using the keywords "Satoyoshi", "Satoyoshi syndrome" or "komuragaeri disease". We included all cases up to December 2019, without limiting year of publication or language. We also explored the references of the OMIM, ORPHANET and Rare diseases web pages. The lists of references from the articles found by electronic search were also reviewed to identify additional records. The results of the search are shown in the flow chart in Fig. 1.

A total of 56 publications describing 67 cases of SS were found; 39 (58.2\%) cases described digestive manifestations [1, 2, 4-8, 11-32] (Table 1). The cases that were described across several publications were counted as one single case, and the data provided by the additional publications were pooled together to complete their description [33-37].

\section{Inclusion criteria}

The cases were considered to have gastrointestinal manifestations if at least one of the following circumstances was present:

- Diarrhea, defined as an increase in the frequency of bowel movements, accompanied by a decrease in their consistency. The presence of abdominal pain was considered when it was not related to the presence of spasms or cramps in the abdominal musculature.

- Positive malabsorption test results, including a positive D-xylose test or other evidence of carbohydrate malabsorption. Data compatible with protein or iron absorption deficit have also been considered. -Histological alterations in any section of the digestive tract. Histological samples were obtained by endoscopy or necropsy.

- Endoscopic and radiological data consistent with digestive involvement or malabsorption, such as atrophy or loss of intestinal folds.

\section{Data extraction}

The following data were extracted from each of the cases:

- Clinical and epidemiological data: age of onset of any of the symptoms pertaining to the syndrome, age of onset of digestive symptoms, sex and country of origin. Presence of diarrhea, number of bowel movements, abdominal pain, bloating, nausea, vomiting, weight loss, short stature or stunted growth, and symptoms of malnutrition or cachexia.

- Analytical data: the presence of anemia, iron deficiency, hypoproteinemia or hypoalbuminemia. Presence of autoantibodies.

- Malabsorption tests: results of the D-xylose Test and other malabsorption tests.

- Results of simple radiology tests, ultrasound, computed tomography, barium intestinal transit, or enema.

- Results of endoscopic examinations: gastroscopy or colonoscopy.

- Histological data obtained through endoscopy, biopsy, and from samples collected during surgery or necropsy.

- Treatments used, evolution and prognosis of digestive symptoms, and data regarding analytical or histological alterations, if available. Morbimortality and sequelae.

\section{Results}

\section{Epidemiological data}

The mean age of presentation of SS was 12.8 years (range, from a few months to 52 years), and the mean age of presentation of digestive symptoms was slightly higher ( 15.08 years, range from a few months to 53 years). Thirty-two patients (82\%) were under 18 years of age at onset of disease. In 10 cases, the digestive symptoms were part of the manifestation of disease onset $[1,2,7,8,13,15,19,31]$, together with muscular spasms. Thirty-two of the patients were women (82\%). Eighteen of these 39 cases (46.15\%) were Japanese patients, although cases have been described worldwide.

\section{Gastrointestinal clinical features}

The most common symptom was diarrhea, present in 36 (92.3\%) cases $[1,2,4-8,11-16,18-32]$. Diarrhea was described as an increase in the number of stools and a decrease in consistency. The diarrhea of patients with SS is of a chronic nature, although not necessarily continuous; in fact, it may present as recurrent episodes [13, 22]. The number of daily stools was between 2 [27] and10 [2]. It was reported that foods with high carbohydrate content could trigger an episode of diarrhea [19]. Moreover, low carbohydrate diets were said to improve 


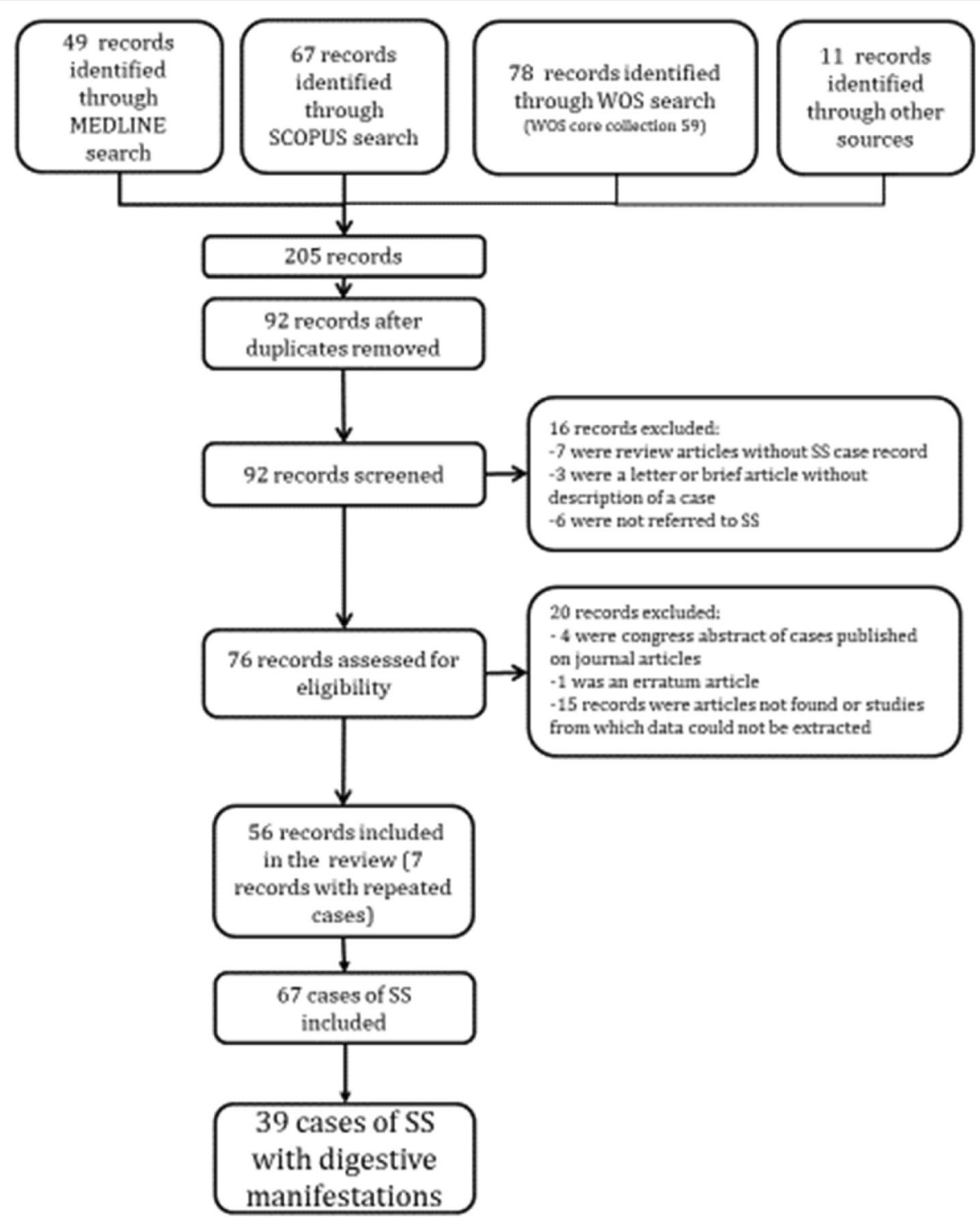

Fig. 1 Flow chart illustrating case selection strategy

diarrhea [2]. Mild steatorrhea was also reported in one case [28]. There were no reports of blood, mucus or pus in the stool.

Other digestive symptoms associated with diarrhea were recurrent abdominal pain (6 cases) $[2,5,7,14,20,21]$, nausea ( 2 cases) $[5,20]$ and vomiting ( 2 cases) $[15,22]$. Weight loss was reported in 9 patients $[1,4,5,11,16,22]$, and short stature or growth retardation in children were reported in 16 cases $[1,2,6,11,14,17-19,23,26,27,29]$. Severe diarrhea with cachexia and dehydration could have led to the deaths of 3 patients $[1,4]$.

\section{Laboratory analyses}

In 26 patients, results of laboratory analyses were provided, such as hemograms or basic biochemistry data $[1,2,4-6,8,11,13-17,19-21,23-30]$. Thirteen patients reported anemia $[1,4-6,11,19,23,26,28-$ $30]$, which was usually microcytic, but hemoglobin levels were only reported in seven patients, and ranged between 8.5 and $12.1 \mathrm{mg} / \mathrm{dl}[4-6,8,26,28$, 30]. In 6 out of 10 cases, there was an explicit mention of iron deficiency $[5,6,11,14,19,30]$. In 7 [1, $4,6,8,23,24,29]$ out of 11 patients [1, 4, 6, 8, 14, $15,23,24,27,29,30]$, hypoproteinemia was described, and in only one [8] of 4 patients $[8,13,15$, 27], hypoalbuminemia was present. Total cholesterol was reported in 11 cases, and its level ranged between $98 \mathrm{mg} / \mathrm{dL}$ and $169 \mathrm{mg} / \mathrm{dL}[1,4-6,24,25,27,28,30]$. In 6 patients, total triglycerides were reported $[5,6$, $8,25,27,28]$, which ranged between 54 and $115 \mathrm{mg} /$ 


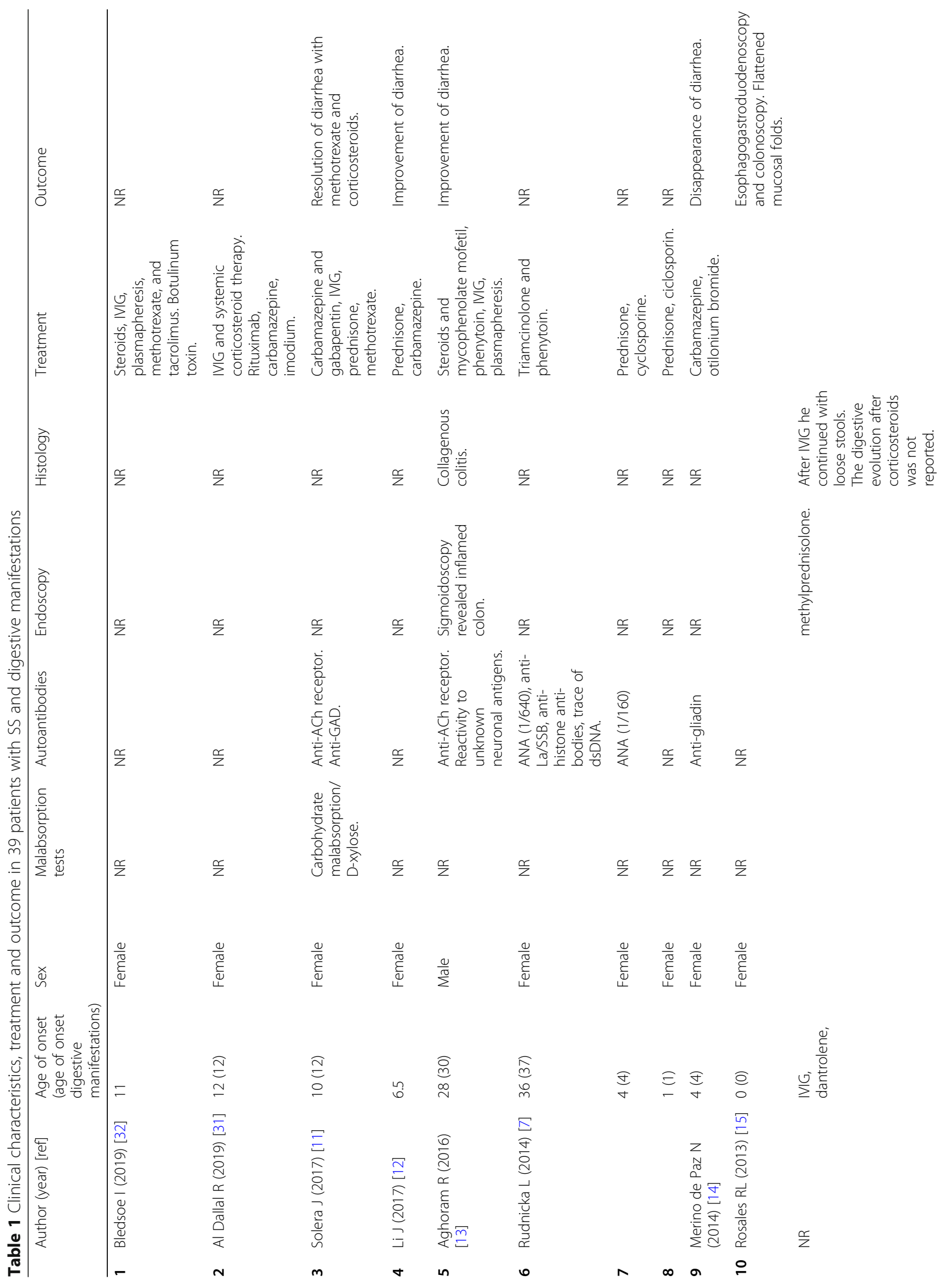




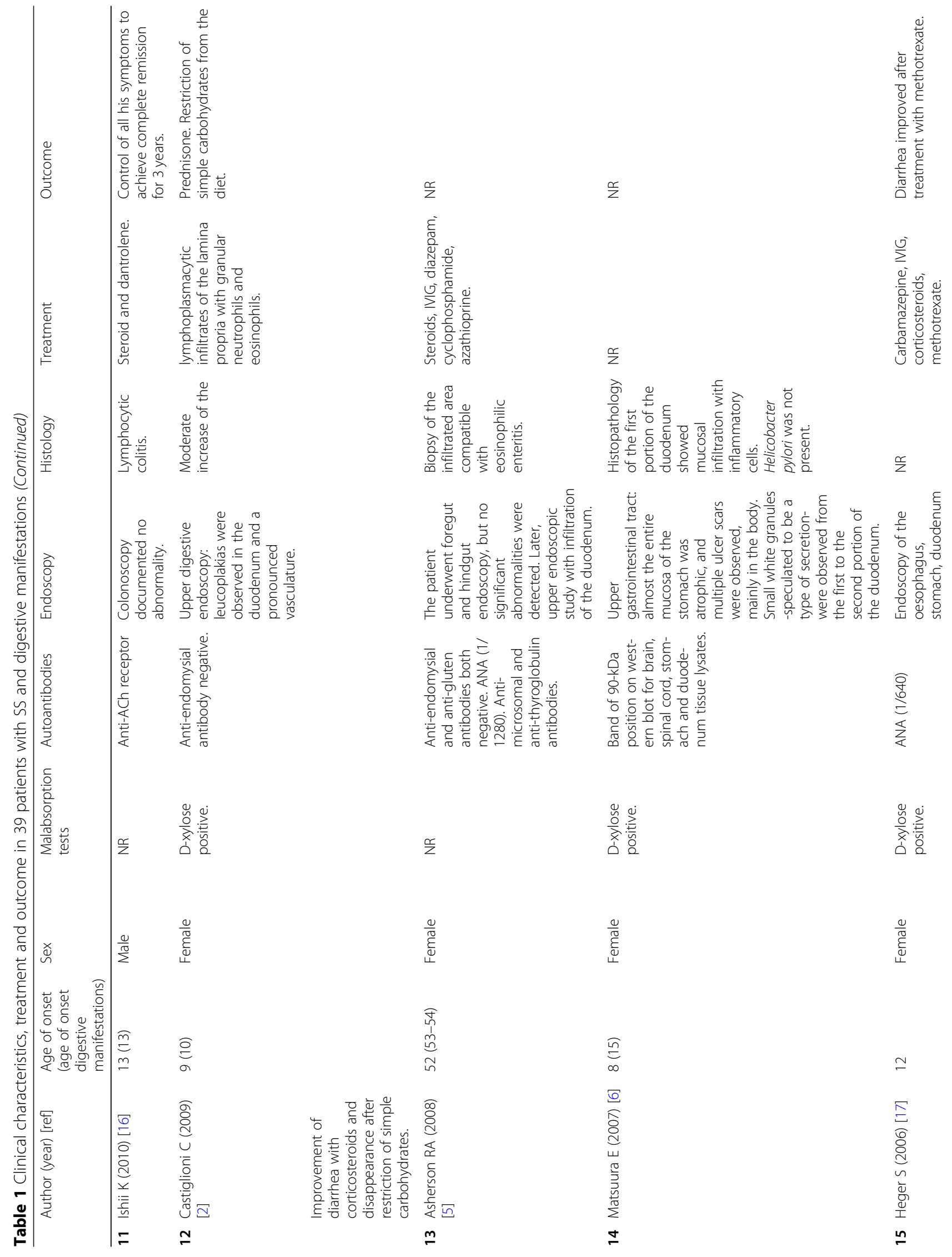




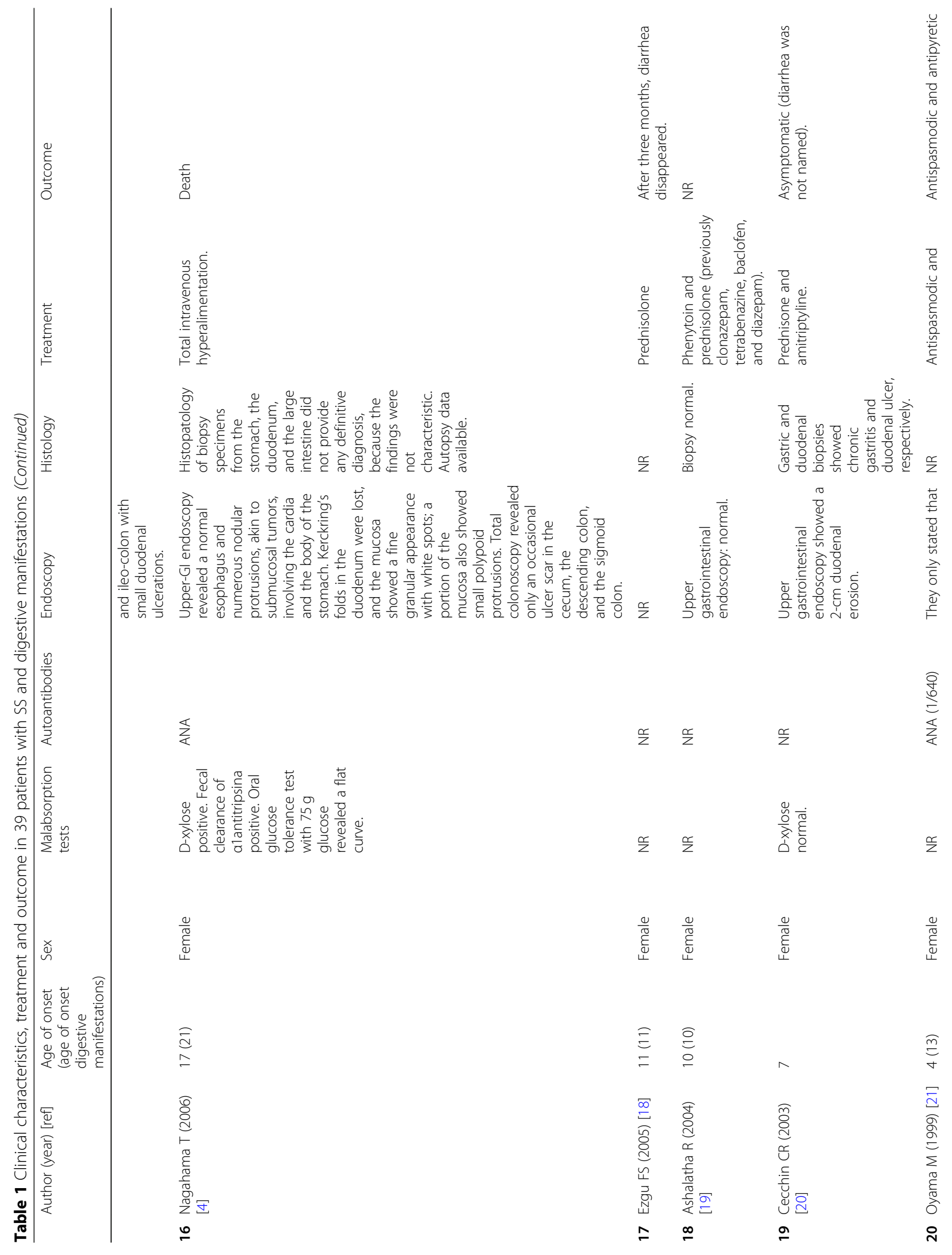




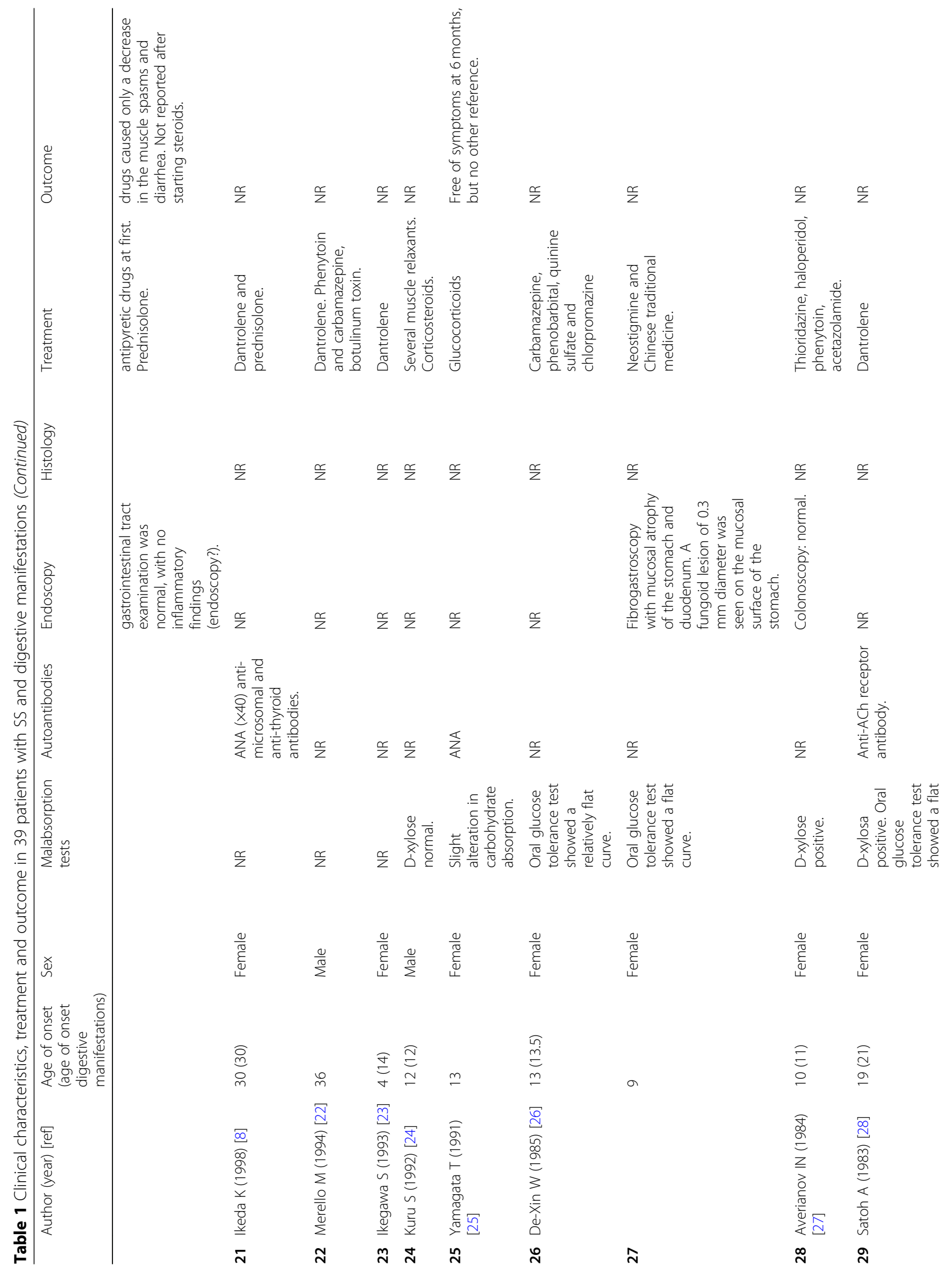




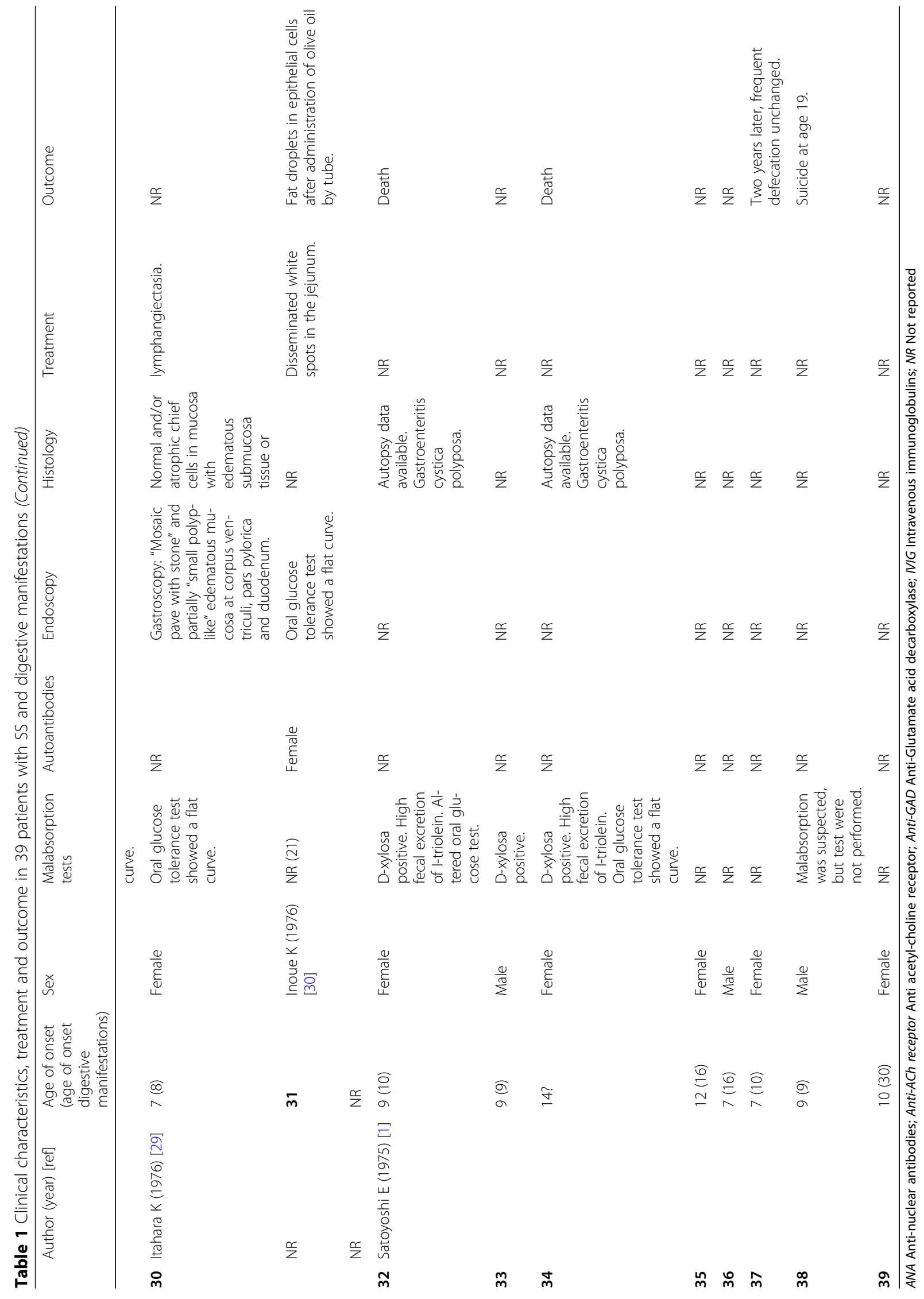


dL. Transaminase levels were normal in all 14 patients for whom they were reported $[1,2,4,5,13,17$, 20, 24-28, 30].

Only one case presented fecal occult blood [24]. In another case, there was no increase in eosinophils or presence of eggs or parasites in feces in the only case in which these parameters were assessed [16].

Carbohydrate malabsorption was assessed by oral glucose tolerance in the first cases published, being progressively replaced in more recent studies by the more accurate D-xylose test. The D-xylose test detected carbohydrate malabsorption in ten out of twelve cases $[1,2,4$, $6,11,17,27,28]$. In one case with a positive D-xylose test, diarrhea was not reported [17]. Two cases of diarrhea with a negative $\mathrm{D}$-xylose test were also reported $[20,24]$. In 13 patients, an oral glucose tolerance test with 50 to $100 \mathrm{~g}$ of glucose was performed [1, 4, 8, 17, $19,24,26,28-30]$. One patient was unable to complete the test due to intolerance [19]. The glucose curve showed a flattened morphology in 9 of the remaining 12 cases, as a manifestation of carbohydrate malabsorption $[1,4,26,28-30]$. In five of these nine patients, both tests (D-xylose test and oral glucose tolerance test) were positive $[1,4,28]$. There was a case with a negative oral glucose tolerance test and a positive D-xylose test [17]. Protein loss through the gastrointestinal tract, measured by alpha-1 antitrypsin clearance, was also described in one patient [4]. This patient also suffered from carbohydrate malabsorption, as demonstrated by both the Dxylose test and the oral glucose tolerance test. The I131-triolein test in feces for fat malabsorption yielded pathological results in two patients [1]. Two cases with increased fat in stool [1] and mild steatorrhea [28] were also found.

Antinuclear antibody determination was positive in 8 $[4,5,7,8,17,21,25]$ out of 16 cases $[4,5,7,8,11,13-$ $17,19-21,24,25]$. The title oscillated between $1 / 40$ and $1 / 1280[5,7,8,17,21]$. In three cases, the pattern of antinuclear antibodies was speckled [5, 7, 25]; one of these cases had SSB antibodies [7]. Other autoantibody tests for SSA, anti-RNP and anti-SM were performed in 4 patients $[6,11,21,25]$, but the results were negative. There was no reference to the antibody pattern in the remaining five patients. In one patient, anti-gliadin antibodies were detected [14]. In two other cases, antiendomysial antibodies were negative $[2,5]$. No antitransglutaminase antibodies were reported in any patient. Anti-acetylcholine receptor antibodies were observed in four $[11,13,16,28]$ out of eight patients $[6,8$, $11,13-16,28]$. Other autoantibodies detected included: anti-thyroid antibodies in two $[5,8]$ out of 6 patients [5, $8,13,15,17,28]$, anti-GAD in one [11] out of 5 patients [5, 11, 14-16], anti-histone antibodies in one case [7], and reactivity against unknown neuronal antigens in another one [13]. Endo et al. [38] detected serum autoantibodies on brain tissue lysates using the western blot technique in a SS patient. Matsuura et al. described the case of a patient with SS and diarrhea, for whom a western blot was performed by incubating patient serum with different tissue lysates. They detected a band at the 90-kDa position for brain, stomach and duodenum tissue lysates, but not for spinal cord and uterine tissue lysates. Furthermore, the authors found the same autoantibodies in another patient in this study [6]. We have also recently confirmed the presence of those same autoantibodies detected by Matsuura et al. in the serum of a Spanish patient [11, 33, 34], using western blot for human brain, stomach and duodenum tissue lysates (unpublished data). Moreover, Aghoram et al. detected nonspecific immune reactivity in a patient's serum exposed to monkey cerebellum and peripheral nerve tissue using indirect immunoflourescence [13].

\section{Radiological features}

Abdominal radiological tests were described in 9 cases $[1,4,6,13,15,19,27]$ (Table 1 ). In only two cases, do the authors comment on simple radiology studies. Nagahama described radiological findings in the small intestine, with loss of Kerckring's folds throughout [4]. Ashalatha described abdominal ultrasound and abdominal CT, without pathological findings in the digestive tract [19]. Aghoram also reported a normal abdominal CT in a 30 year-old man with SS [13].

Five patients underwent oral barium studies $[1,6,27]$ and in two cases, had barium enemas [1]. Aver'ianov reported a 13-year old girl who had a normal oral barium study [27]. Satoyoshi reported 3 cases in which oral barium studies were normal in the early stages; however, in later stages, two of these three cases yielded abnormal barium results [1]. This same author reported two more patients with barium enemas that were normal in the early stages, but abnormal in one of the patients at a later stage [1]. Matsuura, with a double contrast oral barium study, found a mild luminal dilatation and a decrease of Kerckring's folds, which can be interpreted as secondary to chronic inflammatory damage. Matsuura et al. also reported a fine granulation of the second portion of the duodenum and "mesh-like" changes in the mucosa at the end of the second and third portions of the duodenum. An unclear contour and flocculation of the barium was also described, suggesting a malabsorption syndrome [6].

Finally, Ishii performed scintigraphy with a $99 \mathrm{mTc}$ highsolid anaerobic digestion pool in a patient with Satoyoshi that showed an accumulation of isotopes in the colon and small intestine, suggesting leakage of albu$\min [16]$. 


\section{Endoscopic findings}

Sixteen patients underwent digestive endoscopy [2, 4-6, $8,13,15-17,19-21,26,27,29,30]$. Of these, 13 patients received an upper digestive endoscopy $[2,4-6,8,15,17$, $19-21,26,29,30]$ and in 8 cases, a colonoscopy $[4,5,8$, $15,13,16,17,27]$. In one patient, two upper gastrointestinal endoscopies were performed. The main endoscopic findings are reported in Table 1.

In one case, the endoscopy was macroscopically normal, although histological alterations were found [16]. In another patient, the endoscopic abnormal findings were discovered following a second endoscopic study [5]. Endoscopic findings included: atrophy of the stomach mucosa, with multiple ulcer scars mainly in the gastric body together with whitish granules from the first to the second portion of the duodenum [6], gastric or duodenal ulcerations [6, 17, 20], duodenum leukoplakia [2] and areas of duodenal mucosal infiltration [5]. Colonoscopy findings were: inflammation [13], flattening of mucous folds [15] and ulcerations [4]. Five colonoscopies were normal $[5,8,16,17,27]$. A characteristic endoscopic finding described by Nagahama et al. was the presence of nodular protrusions similar to submucosal tumors that can affect different parts of the digestive tract, including the cardia, body of the stomach and duodenum [4]. Using endoscopic ultrasonography, these protrusions appeared as cystic lesions in the stomach wall [4] compatible with the lesions of gastritis cystic polyposa, the histology of which is described below.

\section{Histological features}

Data on the histology of the gastrointestinal lesions were described for 11 patients [1, 2, 4-6, 13, 16, 19, 20, 29] (Table 1). In three of these cases [1, 4], the histological description came from autopsy examination. In 7 patients, the samples were obtained during an upper digestive endoscopy $[2,4-6,19,20,29]$. In three cases, the data came from biopsies extracted during a colonoscopy $[4,13,16]$. In one patient, the histological findings were derived from colonoscopy, upper gastrointestinal endoscopy and autopsy [4].

\section{1. - Biopsies from upper gastrointestinal endoscopy:} There was a broad spectrum of histological manifestations, including normal biopsies [19] or unspecific findings [4]. The most frequent finding was an inflammatory infiltrate $[2,5,6]$, predominantly lymphoplasmacytic [2], although eosinophilic infiltrate was also described [5]. Atrophy of chief cells and edematous submucosal tissue in the stomach was found in one case [29]. Findings compatible with chronic gastritis [20] were reported in one patient.
2. -Colon biopsies: Ishii et al. reported findings compatible with a diagnosis of lymphocytic colitis [16]. Aghoram et al. described findings suggestive of collagenous colitis [13].. Nagahama et al. reported that histopathologic examinations did not yield characteristic findings [4].

3. -Autopsy findings: Data from 3 autopsies were described. Satoyoshi reported two cases of SS with autopsy data [1], and Nagahama et al. described another one [4]. The average time elapsed between the age of symptom onset and the time of necropsy in these three patients was 18.6 years. In the rest of the patients, the time from onset of symptoms to biopsy was 7.7 years. Autopsy data revealed alterations throughout the gastrointestinal tract. Infiltration of gastric and duodenal mucosa by predominantly lymphoplasmacytic cells was described in all cases $[1,4]$. This infiltration of the mucosa also affected the small intestine and the colon. Together with the inflammatory infiltrate, fibrosis of the mucosa and thickening of the muscularis mucosa were present. A characteristic finding in all three cases was the appearance of cystic lesions throughout the gastrointestinal tract, corresponding to glandular cystic dilations containing PAS-positive material. Ulcerative lesions were also found, penetrating the muscularis mucosa in the rectum [1].

\section{Treatment and outcome}

Data regarding treatment were present in 28 of the cases with digestive symptoms $[2,4,5,7,8,11-28,31,32]$. However, outcome in terms of gastrointestinal symptoms was described in only 12 [1, 2, 4, 11-18, 21].

In one patient, the treatment administered was hyperalimentation iv, but diarrhea did not improve [4]. In another patient, different treatments were prescribed, including phenytoin, carbamazepine, immunoglobulins and dantrolene, but likewise, diarrhea did not improve [15]. After that, this patient began corticosteroid treatment, but there is no reference as to whether this measure improved loose stools [15]. One patient improved with carbamazepine and otilonium bromide treatment [14]. Oyama et al. reported that in their patient, antispasmodic and antipyretic drugs only caused a slight decrease in diarrhea; however, outcome after beginning corticosteroid therapy was not described [21].

In 6 patients, diarrhea improved with a treatment regimen that included corticosteroids, alone or in combination $[2,11-13,16,18]$. In addition to these six patients, two other cases that were "asymptomatic" without express reference to digestive manifestations, had also received corticosteroid therapy $[20,25]$. Treatment with corticosteroids or immunosuppressants can cause remission of the 
whole symptomatic spectrum of SS. However, in one of the patients that improved with corticosteroids, diarrhea improved further after including a low-carbohydrate diet in the treatment plan [2]. Another patient improved by adding methotrexate after corticosteroids failed to control diarrhea [17]. Complete remission of diarrhea for at least 3 years was reported with corticosteroid treatment [16].

Four patients died, three of them due to the poor evolution of the disease $[1,4]$. Two of them had severe malnutrition and uncontrolled diarrhea. They developed calcium and ionic disturbances. Both patients died after an episode of seizures followed by coma [1]. A third patient was treated with intravenous hyperalimentation due to diarrhea and malabsorption to improve her nutritional status. She also had episodes of acute pancreatitis. One year later, she underwent surgery consisting of gastrojejunostomy, percutaneous enterostomy, and percutaneous cholangiostomy. A few months after surgery, the patient died of sepsis with multiple organ failure [4]. The fourth patient committed suicide ten years after the onset of the disease [1]. It is not reported that these patients received corticosteroids or immunosuppressants.

\section{Discussion}

As demonstrated in our review, more than half of the patients described with SS have digestive manifestations, of which the main symptom is diarrhea. The clinical picture of these patients can vary from practically asymptomatic with a positive $\mathrm{D}$-xylose test, to very symptomatic patients with more severe diarrhea and signs of malabsorption, cachexia and dehydration. Diarrhea in SS can lead to significant consequences for the patient, including malnutrition, growth retardation, interference with daily life activities and even death. Diarrhea in SS is usually noninflammatory, without blood or mucus in the stool. The D-xylose test appears to be more accurate in determining carbohydrate malabsorption; therefore it has been replacing the oral glucose tolerance test used mainly in the first published cases.

Most of the radiological tests performed showed nonpathological or non-specific findings. Endoscopic explorations often revealed alterations in the gastrointestinal mucosa, such as infiltrative and granular mucosa or ulcers in stomach, duodenum and colon. Histological data showed a predominantly lymphoplasmacytic inflammatory infiltrate that can involve all sections of the digestive tract. In the 3 patients who underwent autopsy, cystic lesions suggestive of gastritis cystica polyposa were discovered. This variation in lesions, ranging from a mild inflammatory infiltrate in the mucosa to more severe lesions with atrophy, fibrosis and the appearance of cystic lesions, suggests a progressive disease course. Moreover, autopsy patients showed more severe lesions. The time elapsed between the age of symptom onset and necropsy/biopsy was greater (18.6 years) in these patients than for the other subjects (7.7 years).

The combination of diarrhea with muscle spasms, cramps, alopecia and osteoarticular deformities is very specific to SS [1]. However, SS may mimic other diseases with non-inflammatory diarrhea, weight loss, growth retardation and signs and symptoms of malabsorption, including: celiac disease, refractory sprue, tropical sprue, Whipple's disease, drug-induced colitis, autoimmune enteropathy, amyloidosis or inflammatory bowel disease $[39,40]$.

In terms of the differential diagnosis (Table 2) of SS, celiac disease and SS have many overlapping symptoms. For example, they both present manifestations secondary to nutrient malabsorption, with diarrhea, weight loss or iron-deficiency anemia. Both diseases also predominate in women in the first decades of life. Additionally, celiac disease can be associated with other conditions described in SS patients, such as autoimmune thyroid disease or myasthenia gravis [40]; even the rare manifestations of celiac disease, such as infertility, arthralgia, arthropathy or alopecia are part of the clinical spectrum of SS [40]. However, the improvement of symptoms with a gluten-free diet favors a celiac disease diagnosis, although this diet has not been tested in patients with SS. On the other hand, the appearance of muscle cramps points towards the diagnosis of SS. In terms of laboratory analysis, anti-gliadin antibodies present in celiac disease have been detected in one case of SS. Anti-endomysial antibodies also common to celiac disease were assessed in two SS cases, but with negative results. Anti-transglutaminase antibodies have not yet been tested for in SS. Histologically, the inflammatory cells in intestinal biopsies characteristic of celiac disease are also common to SS and may lead to an initial diagnosis of celiac disease in SS patients. In the future, detailed characterization of intraepithelial lymphocytes may provide important distinguishing features for Satoyoshi patients.

As in SS, inflammatory bowel disease can also manifest in young patients with diarrhea and weight loss. However, signs of inflammatory diarrhea, proctitis, bloody diarrhea, tenesmus, perianal fistulas and stenotic or fibrotic alterations do not occur in patients with SS. Arthritis can be present in inflammatory bowel disease, mainly in Crohn's disease in the form of migratory and asymmetric polyarthritis; in SS, however, the most frequent osteoarticular manifestations are bone deformities and alterations in the metaphysis, but not arthritis.

With respect to irritable bowel syndrome, there may be increased frequency of bowel movements, but this syndrome usually involves abdominal pain and distension, which are less frequent in SS. In addition, irritable bowel syndrome does not show signs or symptoms related to the malabsorption, weight loss or malnutrition of SS. 
Table 2 Differential diagnosis of diarrhea caused by Satoyoshi syndrome

\begin{tabular}{|c|c|c|}
\hline & Similarities with Satoyoshi syndrome & Differences with Satoyoshi syndrome \\
\hline Celiac sprue & $\begin{array}{l}\text { Most frequent in women in the first decades of life. } \\
\text { Diarrhea with nutrient malabsorption } \\
\text { Immune basis } \\
\text { Iron deficiency anemia } \\
\text { Weight loss } \\
\text { Associated with other conditions such as autoimmune } \\
\text { thyroid disease or myasthenia gravis. It can associate } \\
\text { with rare manifestations such as infertility, arthralgia, } \\
\text { arthropathy or alopecia. } \\
\text { Intestinal biopsies with inflammatory infiltrate }\end{array}$ & $\begin{array}{l}\text { Improvement with gluten free diet. Muscle spasm or } \\
\text { cramps not present }\end{array}$ \\
\hline Drug induced Diarrhea & $\begin{array}{l}\text { Diarrhea Duodenitis with inflammatory infiltrate in } \\
\text { biopsies }\end{array}$ & $\begin{array}{l}\text { Improvement after drug withdrawal Muscle spasm and } \\
\text { alopecia are not present }\end{array}$ \\
\hline $\begin{array}{l}\text { Inflammatory bowel } \\
\text { disease }\end{array}$ & $\begin{array}{l}\text { Diarrhea and weight loss It can be present in young } \\
\text { patients }\end{array}$ & $\begin{array}{l}\text { Inflammatory diarrhea, proctitis, bloody diarrhea, tenesmus, } \\
\text { perianal fistulas, stenotic or fibrotic alterations osteoarticular } \\
\text { involvement in form of migratory and asymmetric polyarthritis. } \\
\text { In SS, bone deformities and alterations in the metaphysis are } \\
\text { more frequent. }\end{array}$ \\
\hline Irritable bowel syndrome & Increased frequency of bowel movements & $\begin{array}{l}\text { No signs of malabsorption or malnutrition. Associated with } \\
\text { fibromyalgia, chronic fatigue syndrome, chronic back pain, } \\
\text { chronic pelvic pain, chronic headache, and temporomandibular } \\
\text { joint dysfunction, depression, anxiety }\end{array}$ \\
\hline Autoimmune enteropathy & $\begin{array}{l}\text { Immune-mediated intestinal mucosal atrophy can be } \\
\text { cause of watery diarrhea }\end{array}$ & $\begin{array}{l}\text { Alopecia, muscle spasms and skeletal alterations are nor } \\
\text { present in autoimmune enteropathy }\end{array}$ \\
\hline
\end{tabular}

Autoimmune enteropathy, a rare condition that can occur both in children and adults, must also be differentiated from SS. It is characterized by immune-mediated intestinal mucosal atrophy that can affect the esophagus, stomach, small bowel and colon. However, it is the clinical picture of SS, with alopecia, muscle spasms and skeletal alterations that differentiates it from autoimmune enteropathy.

Medications, such as nonsteroidal anti-inflammatory drugs (NSAID) or olmesartan, can also cause diarrhea and duodenitis. NSAID duodenitis presents with histological alterations in the duodenum, reminiscent of those of celiac disease. These lesions consist of a non-specific infiltration of the neutrophil and plasma cells in lamina propria, with intraepithelial lymphocytes and low-grade villous blunting. Furthermore, prolonged use of these anti-inflammatory drugs can cause iron-deficiency anemia. On the other hand, in some patients, olmesartan can induce severe diarrhea associated with duodenal inflammation indistinguishable from other pathologies, such as celiac disease. Improvement after drug withdrawal, as well as the other manifestations of SS, can help in the differential diagnosis. The use of these drugs is not described in the patients included in this review.

The treatment of diarrhea does not differ from that of the other clinical manifestations in SS. Corticosteroids and immunosuppressants remain the treatment of choice for diarrhea in patients with SS. Although there is not much data in the follow-up of these patients, at least one case has been documented in which improvement was maintained throughout 3 years of follow-up [16].

The main limitation of our review is that it is composed of isolated cases. Besides, symptom descriptions lack detail. The authors refer to the presence of diarrhea in the majority of cases; however, this symptom is poorly characterized. On the other hand, the most complete histological data come from autopsies published many years ago and performed on SS patients who were not treated with immunosuppressants.

Findings such as the presence of specific antibodies against brain, stomach and intestinal lysates have been reported by two authors $[6,38]$. These findings are consistent with the autoimmune character of SS and could be useful in the future as a diagnostic tool. More studies are needed to clarify the role of these antibodies in both the pathogenesis of the syndrome and the diagnostic approach to patients.

\section{Conclusions}

Chronic diarrhea with malabsorption is one of the most disabling symptoms in SS. The clinical spectrum of these patients can vary from practically asymptomatic with a positive D-xylose test, to very symptomatic patients with severe diarrhea. Thus, malabsorption testing seems advisable in presumed SS patients, even if digestive symptoms are absent. Corticosteroids and immunosuppressants are still the treatment of choice for diarrhea in these patients. SS shares clinical manifestations with celiac disease and other autoimmune and gastrointestinal inflammatory syndromes, thus differential diagnosis is key. The recognition of SS is essential for early immunosuppressive treatment and for achieving a favorable outcome. In this regard, determining the specific auto-antibody pattern for SS remains a pending issue for future research in this field. 


\section{Abbreviations}

ANA: Anti-nuclear antibodies; Anti-ACh receptor: Anti acetyl-choline receptor; Anti-GAD: Anti-glutamate acid decarboxylase; IVIG: Intravenous immunoglobulins; NR: Not reported; SS: Satoyoshi syndrome; OMIM: Online mendelian inheritance in man

\section{Acknowledgements}

We thank Alexandra L. Salewski, MSc, for the English revision of the manuscript.

\section{Authors' contributions}

JS, JSGP and CdC conceived and designed the work. JSGP and CdC did the literature search and collected data. JSGP, CdC and JS revised and analyzed data. JSGP, CdC and JS drafted and revised the final manuscript and approved the submitted version.

\section{Funding}

This paper was not funded.

\section{Availability of data and materials}

Not applicable.

\section{Ethics approval and consent to participate}

Not applicable.

\section{Consent for publication}

Not applicable.

\section{Competing interests}

The authors have no relevant affiliations or financial involvement with any organization or entity with a financial interest in or financial conflict with the subject matter or materials discussed in the manuscript. This includes employment, consultations, honoraria, stock ownership or options, expert testimony, grants or patents received or pending, or royalties.

\section{Author details}

${ }^{1}$ Department of Internal Medicine, Complejo Hospitalario Universitario de Albacete, Albacete, Spain. ${ }^{2}$ Department of Medical Sciences, Faculty of Medicine, Universidad de Castilla - La Mancha, Albacete, Spain. ${ }^{3}$ Research Department, Neuropsychopharmacology Unit, Complejo Hospitalario Universitario de Albacete, Albacete, Spain. ${ }^{4}$ Hospital General Universitario de Albacete, Unidad de Neuropsicofarmacología, Edificio de Investigación, $3^{a}$ planta c/ Hermanos Falcó, 37 E-02008, Albacete, Spain.

Received: 4 March 2020 Accepted: 4 May 2020

Published online: 19 May 2020

\section{References}

1. Satoyoshi E. A syndrome of progressive muscle spasm, alopecia and diarrhea. Neurology. 1978;28:458-71.

2. Castiglioni C, Díaz A, Moënne K, Mericq V, Salvador F, Hernández C. Síndrome de Satoyoshi: Enfermedad multisistémica con respuesta exitosa a tratamiento esteroidal. Rev Méd Chile. 2009;137:542-6.

3. Arita J, Hamano S, Nara T, Maekawa K. Intravenous gammaglobulin therapy of Satoyoshi syndrome. Brain Dev. 1996;18:409-11.

4. Nagahama T, Kenshi Y, Oishi T, Iwashita A, Hirai F, Yao T, Matsui T, Takaki Y. GI manifestations of Satoyoshi's syndrome. Gastrointest Endosc. 2006;64: 143-5.

5. Asherson RA, Giampaolo D, Strimling M. A case of adult-onset Satoyoshi syndrome with gastric ulceration and eosinophilic enteritis. Nat Clin Pract Rheumatol. 2008:4:439-44.

6. Matsuura E, Matsuyama W, Sameshima T, Arimura K. Satoyoshi syndrome has antibody against brain and gastrointestinal tissue. Muscle Nerve. 2007; 36(3):400-3.

7. Rudnicka L, Kwiatkowska M, Rakowska A, Czuwara J, Olszewska M. Alopecia areata. How not to miss Satoyoshi syndrome? J Dermatol. 41:2014, 951-956.

8. Ikeda K, Satoyoshi E, Kinoshita M, Wakata N, Iwasaki Y. Satoyoshi's syndrome in an adult: a review of the literature of adult onset cases. Intern Med. 1998; 37:784-7.

9. Ikegawa S, Nagano A, Satoyoshi E. Skeletal abnormalities in Satoyoshi's syndrome: a radiographic study of eight cases. Skelet Radiol. 1993;22:321-4.
10. Solís-García Del Pozo J, de Cabo C, Solera J. Treatment of Satoyoshi syndrome: a systematic review. Orphanet J Rare Dis. 2019;14(1):146. https://doi.org/10.1186/s13023-019-1120-7.

11. Solera J, Álvarez S, Botet J, de Cabo C, et al. J Neurol Sci. 2017:379:226-8.

12. Li J, Peng D, Jiang T, Avivi-Arber L. Satoyoshi syndrome with progressive orofacial manifestations: a case history report. Int J Prosthodont. 2017;30:163-7.

13. Aghoram R, Srijithesh PR, Kannoth S. Adult-onset Satoyoshi syndrome and response to plasmapheresis. Ann Indian Acad Neurol. 2016;19(1):131-3.

14. Merino de Paz N, Rodriguez-Martin M, Contreras Ferrer P, Pestana Eliche M, Noda Cabrera A. Satoyoshi Syndrome: a cause of alopecia universalis in association with neurologic and bony abnormalities. Pediatr Dermatol. 2013;30:e22-4.

15. Rosales, RL; Banzon, JE. Painful muscle spasms, twisting, and loose stools: what a combination in an adolescent girl! Movement disorders: unforgettable cases and lessons from the bedside. ISBN: 978-1-9362-87284. 2013. 217-221.

16. Ishii K, Furusho K, Tamaoka A, Morishita Y. Lymphocytic colitis in Satoyoshi Syndrome. S Med J. 2010;103:591.

17. Heger S, Kuester RM, Volk R, Stephani U, Sippell WG. Satoyoshi syndrome: a rare multisystemic disorder requiring systemic and symptomatic treatment. Brain Dev, 2006. 28:300-4.

18. Ezgu FS, Tumer L, Serdaroglu A, Hasanoglu A, Cansu A, Hirfanoglu T, Dalgic B. The co-existence of Satoyoshi syndrome and myoadenylate deaminase deficiency. J Inherit Metab Dis. 2005;28:253.

19. Ashalatha R, Kishore A, Sarada C, Nair MD. Satoyoshi syndrome. Neurol India. 2004;52:94-5.

20. Cecchin CR, Félix TM, Magalhães RB, Furlanetto TW. Satoyoshi Syndrome in a Caucasian girl improved with glucocorticoids. Am J Med Genet A. 2003; 118A:52-4.

21. Oyama M, Imaizumi T, Mitsuhashi Y, Kondo S. Satoyoshi syndrome. Arch Dermatol. 1999;135:91-2.

22. Merello M, Garcia H, Nogués M, Leiguarda R. Masticatory muscle spasm in anon-Japanese patient with Satoyoshi Syndrome successfully treated with botulinum toxin. Mov Disord. 1994;9:104-5.

23. Ikegawa S, Nagano A, Nakamura K, Kurokawa T. A case of Satoyoshi's Syndrome. J Pediatr Orthop. 13:1993, 793-6.

24. Kuru S, Riku S, Nakayabu M, Kobayashi Y, leda T. A case of 'syndrome of progressive muscle spasm, alopecia, and diarrhea (Satoyoshi)' treated with steroid pulse therapy. Clin Neurol. 1992;32(6):612-5.

25. Yamagata T, Miyao M, Momoi M, Matsumoto S, Yanagisawa M. A case of generalized komuragaeri disease (Satoyoshi disease) treated with glucocorticoid. Clin Neurol. 1991;31:79-83.

26. De-Xin W, Hui-di F. Three cases of recurrent generalized muscle spasm in China. Jap J Med. 1985;24:263-8.

27. Aver'ianov IN, Vodolagin VD, Logunova LV, Lla L. Positive therapeutic effect of diacarb in the syndrome of progressive muscle spasms, alopecia and diarrhea (Satoyoshi syndrome). Zh Nevropatol Psikhiatr Im S S Korsakova. 1984:84:1623-7.

28. Satoh A, Tsujihata M, Yoshimura T, Mori M, Nagataki S. Myasthenia gravis associated with Satoyoshi syndrome: muscle cramps, alopecia and diarrhea. Neurology. 1983;33:1209-11.

29. Itahara K, Oyama K, Ohara Y, Kobayashi K, Tsumuraya K, lijima K, Nakamura S, Rikimaru S. Gastroscopic findings of a patient with recurrent muscle spasm of central origin (Satoyoshi). Clin Neurol. 1976;20:126-8.

30. Inoue K. General muscle crapms (Komuragaeri disease) and its intestinal absortion. Clin Neurol. 1976;20:699-705.

31. Al-Dallal R, Khan S, Guttenberg K, Shah N. SAT-214 Satoyoshi syndrome: a rare cause of premature ovarian failure. J Endocr Soc. 2019;3(Suppl 1):SAT-214.

32. Bledsoe I. Botulinum toxin treatment of muscle spasm in a case of Satoyoshi syndrome. Mov Dis. 2019;34(suppl 2):S279.

33. Solera J, Rallo B, Herranz AS, Pardal JM, Martin-del Rio R, de Cabo C. High glycine levels in the cerebrospinal fluid in Satoyoshi syndrome. J Neurol Sci. 2015;357(1-2):312-3.

34. Pardal-Fernández JM, Solera-Santos J, Iniesta-López I, Rodríguez-Vázquez M. Satoyoshi's syndrome related muscle spasms: functional study. Rev Neurol (Paris). 2012;168(3):291-5.

35. Li J, Jiang T, Feng HL. A case report of dental abnormality and prosthetic treatment of Satoyoshi syndrome. Zhonghua Kou Qiang Yi XueZaZhi. 2008; 43(4):213-5.

36. Satoh A, Yoshimura T, Mori M, Tsujihata M, Takamori M. A case of generalized komuragaeri disease complicating myasthenia gravis (author's transl). Rinsho Shinkeigaku. 1982;22(3):251-7. 
37. Satoyoshi E, Yamada K. Recurrent muscle spasm of central origin. A report of two cases. Arch Neurol. 1967;16:254-64.

38. Endo K, Kumagai T, Nakahara T, Nakamura K, Shimizu M, Watanabe A, et al. A novel autoantibody associated with Satoyoshi sybdrome.

Neuroimmunology (Tokyo). 2001;9:102-3.

39. Owen DR, Owen DA. Celiac disease and other causes of duodenitis. Arch Pathol Lab Med. 2018;142:35-43.

40. Farrell RJ, Kelly CP. Celiac sprue. N Eng J Med. 2002;346:180-8.

\section{Publisher's Note}

Springer Nature remains neutral with regard to jurisdictional claims in published maps and institutional affiliations.

Ready to submit your research? Choose BMC and benefit from:

- fast, convenient online submission

- thorough peer review by experienced researchers in your field

- rapid publication on acceptance

- support for research data, including large and complex data types

- gold Open Access which fosters wider collaboration and increased citations

- maximum visibility for your research: over $100 \mathrm{M}$ website views per year

At $\mathrm{BMC}$, research is always in progress.

Learn more biomedcentral.com/submissions 